\title{
Diffusion tensor MRI correlates with executive dysfunction in patients with ischaemic leukoaraiosis
}

\author{
M O'Sullivan, R G Morris, B Huckstep, D K Jones, S C R Williams, H S Markus
}

J Neurol Neurosurg Psychiatry 2004;75:441-447. doi: 10.1136/jnnp.2003.014910

See end of article for authors' affiliations

Correspondence to: Professor Hugh Markus, Clinical Neuroscience, St George's Hospital Medical School, Cranmer Terrace, London SW17 ORE, UK; h.markus@sghms.ac.uk

Received 18 March 2003 In revised form 9 July 2003 Accepted 11 July 2003
Background: Cerebral small vessel disease is a common cause of vascular dementia. Both discrete lacunar infarcts and more diffuse ischaemic changes, seen as confluent high signal (leukoaraiosis) on T2 weighted magnetic resonance imaging (MRI), occur. However, there is a weak correlation between T2 lesion load and cognitive impairment. Diffusion tensor MRI (DTI) is a new technique that may provide a better index of white matter damage.

Objectives: To determine whether DTI measures are correlated more strongly with cognitive performance than lesion load on T2 weighted images, and whether these correlations are independent of conventional MRI parameters.

Methods: 36 patients with ischaemic leukoaraiosis (leukoaraiosis plus a previous lacunar stroke) and 19 healthy volunteers underwent DTI, conventional MRI, and neuropsychological assessment.

Results: On DTI, diffusivity was increased both within lesions and in normal appearing white matter. Mean diffusivity of normal appearing white matter correlated with full scale IQ $(r=-0.46, p=0.009)$ and tests of executive function. These correlations remained significant after controlling for age, sex, brain volume, and $\mathrm{T} 1 / \mathrm{T} 2$ lesion volumes. No significant correlation was identified between T2 lesion load and IQ or neuropsychological scores. Of conventional measures, brain volume correlated best with cognitive function.

Conclusions: Diffusion tensor measurements correlate better with cognition than conventional MRI measures. They may be useful in monitoring disease progression and as a surrogate marker for treatment trials. The findings support the role of white matter damage and disruption of white matter connections in the pathogenesis of cognitive impairment in cerebral small vessel disease.
1: the USA it is estimated that 430000 people have dementia in the context of a previous stroke, and prevalence estimates for vascular dementia in Europe range from $1.5 \%$ to $16.3 \%$ in elderly people. ${ }^{1}$ Cerebral small vessel disease, in which ischaemia occurs in the territory of small perforating cerebral end arterioles, is a major cause of vascular dementia. Patients with this subtype of vascular dementia have a characteristic neuropsychological profile with particular impairment of executive functions, attention, and mental processing speed. The importance of this subtype of vascular dementia was evident in a recent clinical trial where over $60 \%$ of the participants had small vessel disease. ${ }^{2}$

On neuropathological examination in patients with cerebral small vessel disease, a combination of small regions of focal infarction (lacunar infarction) and more diffuse areas of neuronal loss, demyelination, and gliosis is found. ${ }^{3}$ The neuroimaging correlates-which are best seen on T2 weighted magnetic resonance imaging (MRI)-are discrete focal lesions representing lacunar infarcts, and more diffuse white matter hyperintensity referred to as leukoaraiosis. ${ }^{4}$

Cerebral small vessel disease is itself common, independent of dementia. Lacunar stroke accounts for a quarter of all ischaemic stroke episodes. It is likely that a much larger number of individuals have more subtle cognitive disturbance because of cerebral small vessel disease and leukoaraiosis. This group of patients probably has a high risk of developing dementia, and may be a particularly suitable target for preventive treatment. ${ }^{5}$

As originally defined, "leukoaraiosis" is a purely radiological entity. ${ }^{4}$ In patients with vascular dementia and leukoaraiosis, neuropathological data have shown that the underlying pathology is almost always ischaemic, and on a population level leukoaraiosis most often has an ischaemic basis. $^{6}$ However, because leukoaraiosis can occasionally represent pathologies other than small vessel disease, ${ }^{7}$ one approach that has been applied to define a homogeneous pathological group with underlying small vessel disease is to combine radiological findings with a history of lacunar stroke. The term "ischaemic leukoaraiosis" has been defined as the combination of radiological leukoaraiosis and a previous clinical lacunar stroke. ${ }^{8}$ This concept includes patients not fitting criteria for dementia, who represent a large portion of all patients with small vessel disease, and avoids difficulties introduced by the definition of dementia.

Studies in patient groups with small vessel disease ${ }^{90}$ and in asymptomatic community populations $\mathrm{s}^{11-13}$ have found only relatively weak correlations between lesion load on T2 weighted imaging and the degree of cognitive impairment. The lack of a strong correlation limits the usefulness of T2 weighted MRI in assessing and monitoring disease severity in patients with small vessel disease. One of the difficulties with T2 weighted imaging is that high signal intensity may reflect a wide spectrum of pathological changes, ranging from complete axonal degeneration to relatively benign pathology with preserved fibre architecture. ${ }^{14}$ An attractive hypothesis explaining cognitive impairment in patients with cerebral small vessel disease is that white matter tract damage results in a "disconnection syndrome" cortical-subcortical and cortical-cortical connections. If this

Abbreviations: DTI, diffusion tensor magnetic resonance imaging; MMSE, mini-mental state examination; NAWM, normal appearing white matter; WAIS-R, Wechsler adult intelligence scale-revised; WMS, Wechsler memory scale 
is true, an imaging technique that provides a more direct assessment of the integrity of white matter would be expected to correlate better with cognition, and would be more appropriate for monitoring disease course and progression.

Diffusion tensor MRI (DTI) is a new technique that provides quantitative information about disruption of white matter projections. ${ }^{16}$ It is a further development of diffusion weighted imaging, in which diffusion is measured in at least six non-colinear directions, providing a three dimensional representation of water motion. Axonal membranes and myelin hinder diffusion in white matter so that damage to these structures would be expected to lead to an increase in mean diffusivity, a measure of diffusion averaged in all spatial directions. In addition, in tracts of parallel fibres diffusion occurs preferentially along the direction of the fibres. This directionality of diffusion can be quantified as fractional anisotropy, which varies from zero, where diffusion is equal in all directions, to 1 , where diffusion occurs along a single axis. ${ }^{17}$ Damage to axons or myelin in coherent tracts would be accompanied by a decrease in fractional anisotropy. Measurement of anisotropy makes it possible, for the first time, to obtain maps of white matter tract anatomy. ${ }^{18} \mathrm{~A}$ previous study of patients with lacunar stroke and leukoaraiosis showed both increased diffusivity, consistent with tissue damage, and reduced fractional anisotropy, consistent with disruption of coherent white matter tracts, in the white matter of patients. ${ }^{8}$ Studies in CADASIL, a rare autosomal dominant form of cerebral small vessel disease, have shown a similar pattern. ${ }^{19}$

In addition to improving the assessment of disease severity, DTI may provide new insights into the mechanisms of cognitive dysfunction. The weak correlation between lesion load on T2 weighted images and cognitive performance has been interpreted in two contrasting ways. One view is that damage to white matter tracts does underpin cognitive impairment but that $\mathrm{T} 2$ weighted MRI provides a relatively poor index of white matter damage. Alternatively, some investigators have argued that cognitive function is determined more by changes in perfusion than by structural damage. ${ }^{9}$ By applying better indices of tissue damage, such as DTI, it will be possible to determine the extent to which cognitive dysfunction can be attributed to alterations in white matter structure.

DTI is likely to provide a better index of tissue damage than conventional MRI for two reasons. First, it may provide a quantitative measure of tract damage within T2 weighted lesions, where pathological studies have shown that the severity of fibre damage is highly variable. ${ }^{14}$ Consistent with this, a DTI study in CADASIL found that mean diffusivity within lesions correlated both with disability and with a simple measure of cognitive impairment, the mini-mental state examination (MMSE). ${ }^{19}$ Second, in CADASIL, ${ }^{19}$ and in patients with lacunar stroke and leukoaraiosis, ${ }^{20}$ DTI has revealed abnormalities in white matter that appear normal on conventional MRI sequences. Changes in the normal appearing white matter may also contribute to cognitive function, thereby weakening correlations with lesion burden.

In this study, we assessed the pattern of DTI changesboth within lesions and in normal appearing white matterin a group of patients with ischaemic leukoaraiosis compared with a group of matched control subjects. We then examined the relations between cognitive function and DTI parameters in ischaemic leukoaraiosis. Our aims were, first, to determine whether DTI measures were correlated more strongly with cognitive performance than lesion load on T2 weighted images, and second, to determine whether these correlations were independent of conventional MRI parameters.

\section{METHODS}

\section{Subjects}

Patients with ischaemic leukoaraiosis were recruited from a stroke/transient ischaemic attack (TIA) clinic. Ischaemic leukoaraiosis was defined as diffuse confluent white matter hyperintensity on T2 weighted MRI (fig 1, bottom left) in patients with a history of clinical lacunar stroke. ${ }^{21}$ All patients had a full clinical assessment, ECG, carotid duplex, and MRI scan. Exclusion criteria were: any cause of stroke other than small vessel disease, including large artery stenosis or a cardioembolic source; cortical infarcts on neuroimaging; large subcortical infarcts (more than $15 \mathrm{~mm}$ maximum diameter) as these infarcts often have a large vessel or embolic aetiology; and any history of previous neurological or psychiatric disease. Thirty six consecutive patients were recruited. Twenty four community volunteers, with no current or previous neurological or psychiatric disease, were also recruited as control subjects. Five of these were subsequently excluded from the study when conventional MRI revealed asymptomatic white matter abnormalities.

The groups were matched for age, sex, and educational history. Mean (SD) age was 69.5 (8.8) years (range 50 to 84) in the group with ischaemic leukoaraiosis (ILA group), and 71.6 (7.5) years (56 to 84 ) in the control group $(p=0.35)$. Twenty four $(67 \%)$ of the ILA group were male, compared with $12(63 \%)$ of the control group $(p=0.80)$. Mean (SD) duration of full time education was 10.8 (3.6) years in the ILA group and 11.9 (1.9) years in the control group $(p=0.16)$. Thirty $(83 \%)$ of the ILA group and nine $(47 \%)$ of the control group had a history of hypertension $(p=0.01)$. Four of the ILA group and one of the control group were diabetic.

The local research ethics committee approved the study and subjects gave informed, written consent.

\section{MRI protocol}

MRI was done using a 1.5 T GE Signa MR scanner (General Electric, Milwaukee, Wisconsin, USA).

\section{Conventional imaging}

Dual echo (proton density and T2 weighted), FLAIR (fluid attenuating inversion recovery), and Tl weighted sequences were employed. Slice locations were identical for all of these sequences. Multiple contiguous $3 \mathrm{~mm}$ slices were prescribed in a true axial plane to provide complete brain coverage. Inplane resolution was $0.86 \times 0.86 \mathrm{~mm}$. Acquisition parameters were as follows:

- Dual echo: TE (time of echo) $=17 / 102 \mathrm{~ms}$; TR (time of repetition) $=4000 \mathrm{~ms}$; NEX 1

- FLAIR: $\mathrm{TE}=135 \mathrm{~ms}$; $\mathrm{TR}=9500 \mathrm{~ms}$

- $\mathrm{Tl}$ spin echo: $\mathrm{TE}=20 \mathrm{~ms}$; $\mathrm{TR}=450 \mathrm{~ms}$; NEX 2

Tl weighted images were not obtained for three patients and one control subject.

\section{Diffusion tensor imaging}

A peripherally gated echo-planar imaging sequence, optimised for white matter, was employed, ${ }^{22}$ with $\mathrm{TE}=121.1 \mathrm{~ms}$, $\mathrm{TR}=9 \mathrm{RR}$ intervals on ECG, and maximum strength of diffusion gradients $=22 \mathrm{mT} . \mathrm{m}^{-1}$. In-plane resolution was $1.875 \times 1.875 \mathrm{~mm}$. Between 15 and 18 near axial $5 \mathrm{~mm}$ slices with a $1 \mathrm{~mm}$ gap provided coverage of supratentorial structures in all subjects.

\section{MRI image analysis}

MRI image analysis was undertaken by a single rater blinded to the neuropsychological findings. 


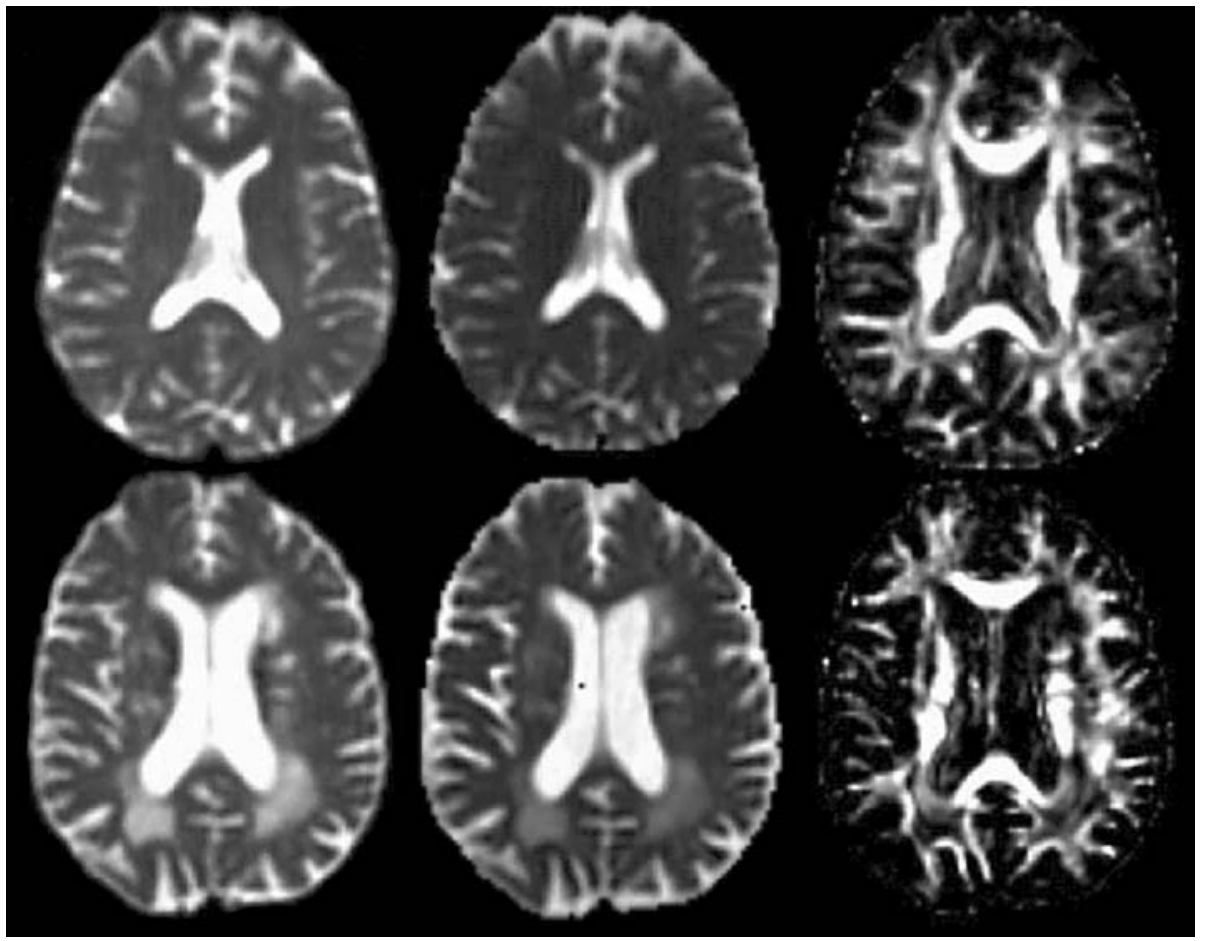

Figure 1 Diffusion tensor magnetic resonance images from a control subject (top) and a patient with ischaemic leukoaraiosis (bottom). T2 weighted images (left), diffusivity maps (centre), and fractional anisotropy maps (right) are shown in identical slice positions for each subject. The fractional anisotropy maps reveal the detailed white matter anatomy and clearly show individual fibre bundles. Increased mean diffusivity and reduced anisotropy are seen in areas affected by leukoaraiosis (bottom). Region of interest analysis also revealed more subtle abnormalities in white matter that appeared normal on the T2 weighted images.

\section{Conventional MRI}

Conventional images were displayed using Analyze (Mayo Clinic, Rochester, Minnesota, USA). Lesion volumes were measured on both Tl weighted and FLAIR. A cursor was placed within each visible lesion and the software then automatically outlined the lesion using fixed intensity thresholds that were the same for all subjects. Areas of diffuse abnormality and discrete lacunar infarcts were included. All regions were checked visually to ensure that they matched the visible extent of lesions. Total lesion volumes were calculated by summing all the areas measured and multiplying by the slice thickness. Total brain volume and the volume of the lateral ventricles were also calculated using a similar intensity threshold approach on the Tl weighted images. Parenchymal volume was calculated by subtracting lateral ventricle volume from whole brain volume.

\section{Diffusion tensor MRI}

In addition to fractional anisotropy and mean diffusivity images, the diffusion tensor imaging sequence produces T2 weighted images with identical slice characteristics (fig 1). To ensure that the observer was not influenced by values of diffusivity or fractional anisotropy, regions of interest (ROIs) were placed on the T2 weighted images. Multiple $5.625 \times 5.625 \mathrm{~mm}(3 \times 3$ voxels $)$ ROIs were positioned. To minimise partial volume effects, ROIs were positioned at least $2 \mathrm{~mm}$ (corresponding to at least one pixel width) from both the edge of the ventricles and the tissue boundaries demarcating abnormal from normal appearing white matter. ROIs were then superimposed in identical positions on mean diffusivity and fractional anisotropy maps, and mean values within them recorded.

Two slices including the anterior horns of the lateral ventricles, and two slices including the posterior horns, were chosen for assessment of periventricular white matter. ROIs were situated in three standardised positions around each horn (fig 2, left). ROIs were included in subsequent analysis only if they were situated entirely within either normal or abnormal appearing white matter, at least $2 \mathrm{~mm}$ from a tissue boundary, and within an imaginary line halfway between ventricle and cortical surface to prevent contamination with cortical grey matter. Two slices through the centrum semiovale were also chosen. For each slice, up to five ROIs were placed in lesion white matter in each hemisphere, and similarly up to five ROIs were placed widely

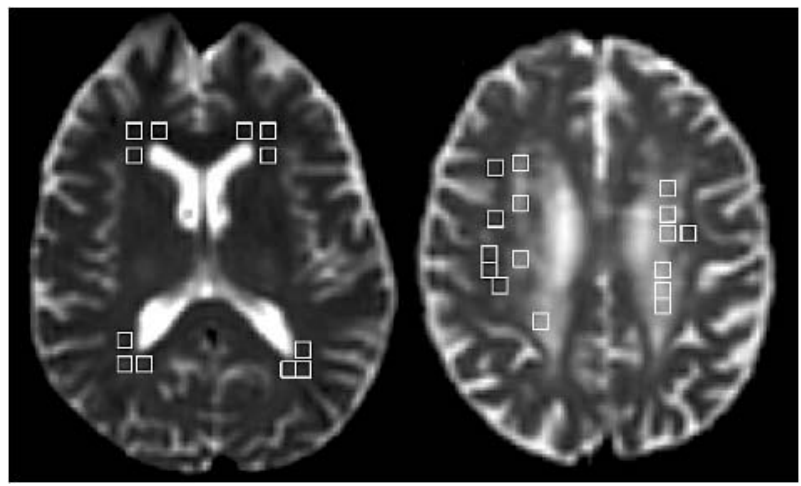

Figure 2 Placement of regions of interest (ROls). Left: positions of ROls in periventricular white matter in a control subject. ROls were placed in these positions on two slices. ROls falling wholly within either a lesion or normal appearing white matter were included in mean measurements. ROls containing both some lesion and some normal appearing white matter were excluded from subsequent analysis. Right: ROls in centrum semiovale white matter in a patient with ischaemic leukoaraiosis. ROls were placed either within lesion or within normal appearing white matter by an operator. Those ROls near to sulci and likely to be contaminated by grey matter were excluded from analysis. 
through normal appearing white matter in each hemisphere (fig 2, right).

\section{Assessment of reliability of measures}

Intrarater reliability was assessed in a randomly selected subgroup of 10 subjects (five patients and five controls). The computer image files were randomised and renamed, and FLAIR, Tl weighted, and DTI images were then reanalysed by the original observer. The within-subject standard deviation has been recommended as a practical index of measurement error. ${ }^{23}$ This was calculated following the method of Bland, and expressed as the within-subject coefficient of variation (within-subject standard deviation as a percentage of the mean value). Coefficients of variation were: T2 weighted lesion volume, $2.9 \%$; parenchymal volume, $3.4 \%$; diffusivity of normal appearing white matter, $1.0 \%$; fractional anisotropy of normal appearing white matter, 5.4\%; diffusivity within lesions, 8.1\%; fractional anisotropy within lesions, $9.7 \%$.

\section{Neuropsychological assessment}

Neuropsychological assessment was done within one week of MRI. One subject suffered a further stroke four days after the MRI and therefore neuropsychological scores were not analysed on that subject. A comprehensive battery of neuropsychological tests was designed, based on a review of relevant published reports. Tests were grouped into cognitive domains, as follows:

General function and global intellectual function: 30 point $\mathrm{MMSE}^{24}$; Wechsler adult intelligence scale-revised (WAISR), ${ }^{25}{ }^{26}$ providing verbal IQ (vocabulary, comprehension, and similarities subtests) and performance IQ (block design and object assembly subtests).

Executive function: Wisconsin card sorting test (WCST) ${ }^{24} 2627$; Reitan trail making 2528 ; verbal fluency ${ }^{25}$; WAIS-R digit symbol; WAIS-R digit span backwards. ${ }^{24}$

Memory: Wechsler memory scale (WMS ${ }^{24-26}$; logical memory and paired associate learning subtests.

Visuospatial function: Benton facial recognition test. ${ }^{12}$

Motor: Finger tapping test.

Administration of the battery took between two and two and a half hours. Two control subjects declined neuropsychological testing, so complete neuropsychological data were available for 17 subjects. Two of the ILA group were unable to perform five and six tests, respectively, because of severe cognitive impairment. One additional subject was unable to do the trail making and Benton facial recognition tests because of severe visual impairment.

\section{Statistical analysis}

To allow direct comparison of different tests, we generated $z$ scores for the neuropsychological measures. These scores were based on the means and standard deviations of each measure in the control group. A $z$ score defines where a score falls in the distribution of scores in normal subjects: a $z$ score of +2.0 corresponds to a score $2 \mathrm{SD}$ above the mean score. Direct comparison of performance between tests was possible because the $z$ scores for all tests were based on an identical control population. MMSE was excluded from this analysis as the scores did not approximate to a normal distribution in control subjects. Group comparisons were made using Student $t$ tests, apart from the MMSE, where a MannWhitney U test was employed.

Correlations between MRI findings and neuropsychological measures were examined in the ILA group only. For each subject, 11 neuropsychological measures were generated so that a large number of possible correlation coefficients and $p$ values could be calculated. However, in this setting Bonferroni correction for multiple comparisons is inappropriate, because the neuropsychological measures are not independent of each other. ${ }^{23}$ When different measures are correlated in this way, a better approach is to undertake a limited number of comparisons using composite measures. Principal components analysis was used to generate a composite score (the first principal component) for cognitive performance. As an initial step in analysis, this composite score was correlated with similar composite scores for DTI of lesions and DTI of normal appearing white matter (giving only two statistical comparisons) to determine whether there was a true underlying relation between DTI findings and cognitive function. The nature of any relation was then explored in more detail using the full range of neuropsychological measures.

\section{RESULTS}

\section{Diffusion tensor imaging: group comparisons}

Table 1 shows the measurements of mean diffusivity and fractional anisotropy in each anatomical region. In the ILA group, differences were found within lesions compared with normal appearing white matter (NAWM) in all anatomical regions. Differences were also found in the NAWM, compared with NAWM of controls, in anterior periventricular white matter and the centrum semiovale.

\section{Pattern of cognitive deficit}

Figure 3 shows mean $z$ score differences between patients and control subjects. In ischaemic leukoaraiosis, performance on measures of executive function was affected more severely than performance in other cognitive domains. Relative preservation of memory performance was also a striking feature. Group differences in timed executive tests (digit symbol and Reitan trail making) remained significant after covarying for motor speed (digit symbol, $\mathrm{p}=0.007$; trail making $\mathrm{B}-\mathrm{A}, \mathrm{p}=0.021$ ).

\section{Correlations between DTI and cognitive function in ischaemic leukoaraiosis}

There was no correlation between lesion load on T2 weighted images and the composite score of cognitive function $(r=0.13, \mathrm{p}=0.49)$. In contrast, there was a significant correlation between the composite score for DTI of normal appearing white matter and cognitive function $(r=0.41$, $\mathrm{p}=0.048$; after covarying for $\mathrm{Tl}$ lesion load, $r=0.53$, $\mathrm{p}=0.013)$. No correlation was found between the composite score for cognition and that for DTI within lesions $(r=0.01$, $\mathrm{p}=0.97)$.

Table 2 shows correlation coefficients for individual tests covarying for age and sex. Diffusivity in the normal appearing white matter of the centrum semiovale correlated both with general intellectual function (full scale IQ) and with more specific measures of executive function (the Wisconsin card sorting test). In the periventricular normal appearing white matter, only total errors on the Wisconsin card sorting test correlated with diffusivity. However, the paucity of normal appearing white matter meant that far fewer measurements were made in the periventricular regions.

Multiple linear regression analyses were done for the three neuropsychological measures with significant univariate correlations with diffusivity: full scale IQ, digit symbol, and both total errors and per cent perseverative errors on the Wisconsin card sorting test. Age, sex, T1 and T2 weighted lesion volume, parenchymal volume, and mean diffusivity of normal appearing white matter were included in a multivariate analysis. For all three neuropsychological measures, only diffusivity of normal appearing white matter remained independently correlated with cognitive performance. The results of multivariate analysis for full scale IQ and Wisconsin card sorting test total errors are shown in table 3.

The multivariate model including mean diffusivity of normal appearing white matter accounted for $52 \%$ of the 
Table 1 Pattern of white matter abnormalities in ischaemic leukoaraiosis

\begin{tabular}{|c|c|c|c|c|c|}
\hline \multirow[b]{2}{*}{ White matter region } & \multicolumn{2}{|c|}{ Ischaemic leukoaraiosis } & \multirow[b]{2}{*}{$\begin{array}{l}\text { Controls } \\
\text { (NAWM) }\end{array}$} & \multicolumn{2}{|c|}{$\begin{array}{l}\text { p Values (ANOVA, post hoc } \\
\text { Tamhane T2) }\end{array}$} \\
\hline & Lesions & NAWM & & $\begin{array}{l}\text { Lesions } v \\
\text { NAWM in ILA }\end{array}$ & $\begin{array}{l}\text { NAWM ILA v } \\
\text { controls }\end{array}$ \\
\hline \multicolumn{6}{|c|}{ Mean diffusivity $\left(\times 10^{-9} \mathrm{~m}^{2} \cdot \mathrm{s}^{-1}\right)$} \\
\hline $\begin{array}{l}\text { Anterior } \\
\text { periventricular } \\
\text { Posterior }\end{array}$ & $1.29(0.20)$ & $0.86(0.04)$ & $0.81(0.04)$ & $<0.001$ & 0.001 \\
\hline $\begin{array}{l}\text { periventricular } \\
\text { Centrum semiovale }\end{array}$ & $\begin{array}{l}1.24(0.16) \\
1.22(0.15)\end{array}$ & $\begin{array}{l}0.83(0.05) \\
0.79(0.04)\end{array}$ & $\begin{array}{l}0.82(0.04) \\
0.75(0.04)\end{array}$ & $\begin{array}{l}<0.001 \\
<0.001\end{array}$ & $\begin{array}{l}0.99 \\
0.001\end{array}$ \\
\hline \multicolumn{6}{|l|}{ Fractional anisotropy } \\
\hline $\begin{array}{l}\text { Anterior } \\
\text { periventricular } \\
\text { Posterior }\end{array}$ & $0.174(0.042)$ & $0.293(0.053)$ & $0.337(0.047)$ & $<0.001$ & 0.011 \\
\hline periventricular & $0.247(0.061)$ & $0.351(0.058)$ & $0.378(0.031)$ & $<0.001$ & 0.128 \\
\hline Centrum semiovale & $0.184(0.034)$ & $0.299(0.031)$ & $0.312(0.024)$ & $<0.001$ & 0.24 \\
\hline \multicolumn{6}{|c|}{$\begin{array}{l}\text { In the ILA group, differences were found within lesions compared with NAWM in all anatomical regions. } \\
\text { Differences were also found in the NAWM, compared with NAWM of controls, in anterior periventricular white } \\
\text { matter and the centrum semiovale. Statistical analysis by analysis of variance with post hoc Tamhane's T2 test for } \\
\text { comparison of pairs. } \\
\text { ANOVA, analysis of variance; ILA, ischaemic leukoaraiosis; NAWM, normal appearing white matter. }\end{array}$} \\
\hline
\end{tabular}

variance in IQ scores, compared with $16 \%$ for a model including age, sex, and lesion loads on $\mathrm{T} 1$ and $\mathrm{T} 2$ weighted images.

\section{DISCUSSION}

This study shows that DTI indices correlate much more strongly with cognitive function than $\mathrm{T} 2$ lesion volume in patients with ischaemic leukoaraiosis. The correlation was strongest for diffusivity of normal appearing white matter, and remained significant after controlling for conventional MRI parameters, including brain parenchymal volume and $\mathrm{Tl}$ and $\mathrm{T} 2$ lesion load. These findings have two important implications. First, they suggest that DTI is a potentially powerful technique for use as a surrogate marker in therapeutic trials. Second, DTI is a promising tool for exploring the mechanisms of cognitive dysfunction in these patients.

In contrast to the positive findings with DTI, no correlations were identified between cognitive function and lesion load measured on $\mathrm{T} 2$ weighted images. This finding is consistent with those of a range of previous studies. Community based studies of healthy older adults have produced inconsistent results, with several early studies reporting no relation between white matter lesions and



Figure 3 The neuropsychological profile found in patients with ischaemic leukoaraiosis. Larger positive scores mean greater impairment. Test measures are grouped by cognitive domain. From left to right: Global intelligence: IQ, full scale IQ. Working memory: Dsback, digit span backwards. Memory: WMS, Wechsler memory scale logical memory; PAL, Wechsler memory scale paired associate learning. Executive: Dsym, digit symbol; FAS, verbal fluency; Trail, Reitan trail making B-A; TE, Wisconsin card sorting test total errors; PE, Wisconsin card sorting test, \% perseverative errors. Visuospatial: Face, Benton facial recognition. cognitive impairment. ${ }^{11}{ }^{12}$ However, recent larger studies have provided more convincing evidence that cognitive function is affected in individuals with white matter hyperintensities. Nevertheless, the relation between lesion burden and the extent of cognitive dysfunction is weak, emerging only in studies that include hundreds of subjects. ${ }^{13}$

One possible factor underlying the lack of correlation in community based studies is that the pathological basis of $\mathrm{T} 2$ weighted hyperintensities is uncertain, and may be heterogeneous. These studies include subjects with minor punctate hyperintensities - data from the Austrian stroke prevention study have shown that these lesions often have a non-ischaemic basis. ${ }^{6}$ This difference in underlying pathology is also reflected in a lack of progression of white matter abnormalities over three years of follow up in those subjects with minor abnormalities at baseline. ${ }^{29}$ In contrast, confluent hyperintensities are most often of ischaemic origin.

An alternative to the population based approach is to study specific subgroups of patients with more advanced disease, in which one might expect stronger correlations between $\mathrm{T} 2$ weighted abnormalities and cognitive dysfunction. All the patients with ischaemic leukoaraiosis in the present study had confluent hyperintensities and had also had a symptomatic stroke; nevertheless, no convincing correlation was identified between $\mathrm{T} 2$ weighted images and cognitive function. Several other studies have adopted clinical and radiological criteria that parallel our definition of ischaemic leukoaraiosis. In these studies, the correlations between $\mathrm{T} 2$ lesion load and cognitive function have also been weak and inconsistent. For example, in a study of 41 patients with cardiovascular risk factors, previous history of stroke, or transient ischaemic attacks and leukoaraiosis, a correlation was found between parts 2 and 3 of the Stroop test and the leukoaraiosis score, but no other correlations were found across a detailed neuropsychological battery. ${ }^{26}$

Of the measures derived from conventional MRI, brain parenchymal volume correlated most strongly with cognitive function. These findings are consistent with previous studies that have shown that cognitive dysfunction correlated with markers of cerebral atrophy such as callosal atrophy $^{30}$ and the volume of the cortical grey matter and hippocampus. ${ }^{10}$ Studies with highly reproducible techniques have revealed a strong relation between atrophy and 
Table 2 Relation between neuropsychological scores and findings from both conventional magnetic resonance imaging and diffusion tensor imaging in the white matter of the centrum semiovale

\begin{tabular}{|c|c|c|c|c|c|c|}
\hline & \multirow{2}{*}{$\begin{array}{l}\text { T2 lesion } \\
\text { volume }\end{array}$} & \multirow{2}{*}{$\begin{array}{l}\text { Parenchymal } \\
\text { volume }\end{array}$} & \multicolumn{2}{|l|}{ NAWM } & \multicolumn{2}{|l|}{ Lesions } \\
\hline & & & MD & FA & MD & FA \\
\hline \multicolumn{7}{|l|}{ General intellectual function } \\
\hline \multicolumn{7}{|l|}{ Executive function } \\
\hline $\begin{array}{l}\text { WCST, total errors } \\
\text { WCST, \% perseverative }\end{array}$ & 0.08 & $-0.38^{*}$ & $0.41^{*}$ & -0.03 & 0.15 & -0.28 \\
\hline errors & -0.01 & $-0.43^{*}$ & $0.43^{*}$ & -0.01 & 0.15 & -0.29 \\
\hline Trail making $\mathrm{B}-\mathrm{A}$ & -0.18 & -0.32 & 0.15 & -0.06 & -0.10 & -0.16 \\
\hline WAIS-R digit symbol & -0.04 & $0.46^{*}$ & -0.29 & 0.08 & -0.09 & 0.23 \\
\hline Verbal fluency (FAS) & 0.20 & 0.04 & -0.18 & 0.04 & 0.31 & -0.10 \\
\hline Digit span, backwards & -0.02 & 0.35 & -0.27 & 0.06 & -0.08 & 0.23 \\
\hline \multicolumn{7}{|l|}{ Memory (WMS) } \\
\hline Logical memory & 0.08 & 0.30 & -0.35 & 0.25 & 0.07 & 0.07 \\
\hline $\begin{array}{l}\text { Paired associate learning } \\
\text { Visuospatial cognition }\end{array}$ & 0.09 & 0.27 & -0.09 & -0.03 & 0.12 & 0.06 \\
\hline Benton facial recognition & 0.03 & 0.12 & -0.22 & 0.21 & -0.10 & 0.15 \\
\hline \multicolumn{7}{|c|}{$\begin{array}{l}\text { Partial correlation coefficients are shown after covarying for age and sex. } \\
{ }^{*} \mathrm{p}<0.05,{ }^{* *} \mathrm{p}<0.01 \text {. } \\
\text { FA, fractional anisotropy; MD, mean diffusivity; WAIS-R, Wechsler adult intelligence scale-revised; WCST, } \\
\text { Wisconsin card sorting test; WMS, Wechsler memory scale. }\end{array}$} \\
\hline
\end{tabular}

cognitive decline in Alzheimer's disease, ${ }^{31}$ and similar rates of atrophy are encountered in patients with vascular dementia (defined by the NINDS-AIREN criteria). ${ }^{32}$ The findings of the present study suggest that measurement of brain atrophy may have a role to play in longitudinal studies in patients with ischaemic leukoaraiosis as well as in other groups.

The observation that visible lesions in T2 weighted images account for only a small proportion of the variance in cognitive performance has led some investigators to argue that the extent of white matter damage is unimportant in determining cognitive function, which is affected by other factors such as hypoperfusion. ${ }^{9}$ The results of this study show that it is important to consider the sensitivity of the technique as an index of white matter damage when assessing the impact of structural changes. While $\mathrm{T} 1$ and $\mathrm{T} 2$ weighted changes accounted for only $16 \%$ of the variance in IQ scores, 52\% could be attributed to white matter damage when better structural indices from diffusion tensor imaging were considered. Our results provide additional evidence that damage to white matter projections is an important mechanism of cognitive impairment, and they are consistent with the "disconnection" hypothesis of cognitive impairment in patients with small vessel disease.

In this study we found that changes in normal appearing white matter correlated with cognitive performance. It is feasible that subjects with a similar extent of white matter hyperintensity on T2 weighted MRI could have different degrees of involvement of the normal appearing white matter, which may be one factor in the relatively weak relation between T2 weighted MRI and cognitive function. In contrast, there was no correlation with measurements within lesions. One possible explanation for this is that relations between neural damage and cognitive dysfunction may be obscured within lesions by changes in glial tissue that also influence patterns of water diffusion. For example, in a lesion within which all axonal projections have already been lost, one would not expect any further change in tissue structure to influence cognitive function. However, diffusivity within this lesion could alter with subsequent changes; loss of glial cells and cavitation would lead to a large increase in diffusivity, while gliosis might further hinder water motion, reducing mean diffusivity. Alternatively, the lack of correlation between DTI measures in lesions and normal appearing

Table 3 Multivariate models for full scale IQ and Wisconsin card sorting test

\begin{tabular}{|c|c|c|c|c|}
\hline & \multicolumn{2}{|c|}{ Univariate } & \multicolumn{2}{|c|}{ Multivariate } \\
\hline & $\bar{R}$ & p Value & $\bar{\beta}$ & p Value \\
\hline \multicolumn{5}{|c|}{ (A) Multivariate model for full scale IQ (WAIS-R) $\left(R^{2}=0.52\right)$} \\
\hline Age & & & 0.438 & $0.011^{*}$ \\
\hline Sex & & & 0.203 & 0.236 \\
\hline Parenchymal volume & 0.47 & $0.009 * *$ & 0.279 & 0.121 \\
\hline T1 lesion load & 0.15 & 0.428 & 0.391 & 0.081 \\
\hline T2 lesion load & 0.01 & 0.977 & -0.128 & 0.542 \\
\hline Diffusivity of NAWM & -0.46 & $0.009^{* *}$ & -0.504 & $0.008^{* *}$ \\
\hline \multicolumn{5}{|c|}{ (B) Multivariate model for Wisconsin card sorting test-total errors $\left(R^{2}=0.39\right)$} \\
\hline Age & & & 0.222 & 0.226 \\
\hline Sex & & & -0.156 & 0.414 \\
\hline Parenchymal volume & -0.38 & $0.043^{*}$ & -0.233 & 0.244 \\
\hline T1 lesion load & -0.03 & 0.891 & 0.008 & 0.974 \\
\hline T2 lesion load & 0.08 & 0.647 & -0.220 & 0.353 \\
\hline Diffusivity of NAWM & 0.41 & $0.020 *$ & 0.413 & $0.046^{*}$ \\
\hline
\end{tabular}


white matter may reflect methodological limitations of the study. Correlations may be easier to demonstrate in normal appearing white matter, simply because this often constitutes the larger portion of the whole white matter, so its measurement provides a more comprehensive measure of damage across the whole brain. Measurements within normal appearing white matter were also more reproducible in the present study. In addition, lesion location is likely to be an important factor in the relation with cognitive dysfunction. Spatial analysis of DTI data has been limited up to now, although the emergence of more sophisticated analysis techniques should provide new insights into the anatomical pattern of correlations with cognitive function. ${ }^{33}$

Relations with cognitive function were weaker for fractional anisotropy than for mean diffusivity. This may reflect the relatively simple analysis techniques used. Diffusivity is relatively constant in white matter, whereas fractional anisotropy values vary widely, depending upon whether the region is placed over the centre of a white matter tract. The position of regions of interest relative to white matter tracts cannot be determined from the $\mathrm{T} 2$ weighted image on which the regions were placed. Therefore small variations in the placement of regions of interest would lead to larger variations in fractional anisotropy than mean diffusivity, as reflected by the reproducibility analysis. With the implementation of more advanced analysis techniques and particularly techniques for highlighting specific tracts (tractography), ${ }^{34}$ correlations with fractional anisotropy are likely to be stronger.

The strong correlation between diffusivity and cognitive function suggests that DTI may be a useful marker of disease severity for use in prospective studies, including treatment trials. An MRI model of disease has several advantages over direct measurement of cognitive function. The cognitive profile of ischaemic leukoaraiosis-as indicated in the present study-is dominated by executive dysfunction. Most simple bedside tools for assessing cognitive function are insensitive to executive dysfunction, ${ }^{35}$ so that direct assessment of cognition is likely to be time consuming. In addition, learning effects can lead to subjects performing better when a test is repeated, which could mask a true decline in cognitive performance. Finally, factors such as mood, cooperation, and motivation can affect the test-retest reliability of direct cognitive assessment.

\section{ACKNOWLEDGEMENTS}

MO'S was supported by a clinical research fellowship awarded by St George's Hospital Medical School. This study was supported by a grant from PPP Healthcare Medical Trust. DKJ was supported by the Wellcome Trust. We thank Dr Paul Summers for help with data acquisition, Dr Mark Horsfield of the Division of Medical Physics, University of Leicester, for providing software for analysis of diffusion tensor images, Professor Martin Bland, St George's Hospital Medical School, for statistical advice, and Dr Chris Clark for reading the manuscript and making suggestions. We also thank Professor Steve Jackson for allowing us to study subjects from the Clinical Age Research Unit database at King's College Hospital, and Emma Ouldred for help with recruiting these subjects.

\section{Authors' affiliations \\ M O'Sullivan, H S Markus, Clinical Neuroscience, St George's Hospital Medical School, London SW17, UK \\ R G Morris, B Huckstep, Department of Neuropsychology, Institute of Psychiatry, London SE5, UK \\ D K Jones, Division of Old Age Psychiatry, Institute of Psychiatry \\ S C R Williams, Department of Neuroimaging, Institute of Psychiatry \\ Competing interests: none declared \\ REFERENCES \\ 1 Roman GC. Vascular dementia: diagnostic criteria for research studies. Report of the NINDS-AIREN International Workshop. Neurology 1993;43:250-60.}

2 Erkinjuntti T, Kurz A, Gauthier S, et al. Efficacy of galantamine in probable vascular dementia and Alzheimer's disease combined with cerebrovascular disease: a randomised trial. Lancet 2002;359:1283-90.

3 Babikian V, Ropper AH. Binswanger's disease: a review. Stroke 1987; 18:2-12.

4 Hachinski VC, Potter P, Merskey H. Leuko-araiosis. Arch Neurol 1987;44:21-3.

5 Erkinjuntti T, Inzitari D, Pantoni L, et al. Limitations of clinical criteria for the diagnosis of vascular dementia in clinical trials. Is a focus on subcortical vascular dementia a solution? Ann NY Acad Sci 2000;903:262-72.

6 Fazekas F, Kleinert R, Offenbacher $\mathrm{H}$, et al. Pathologic correlates of incidental MRI white matter signal hyperintensities. Neurology 1993;43:1683-9.

7 Lotz PR, Ballinger WE, Quisling RG. Subcortical arteriosclerotic encephalopathy: CT spectrum and pathologic correlation. Am J Neuroradiol 1986;7:817-22.

8 Jones DK, Lythgoe D, Horsfield MA, et al. Characterisation of white matter damage in ischaemic leukoaraiosis with diffusion tensor magnetic resonance imaging. Stroke 1999;30:393-7.

9 Sabri O, Ringelstein EB, Hellwig D, et al. Neuropsychological impairment correlates with hypoperfusion and hypometabolism but not with severity of white matter lesions on MRI in patients with cerebral microangiopathy. Stroke 1999;30:556-66

10 Mungas D, Jagust WJ, Reed BR, et al. MRI predictors of cognition in subcortical ischemic vascular disease and Alzheimer's disease. Neurology 2001;57:2229-35

11 Hunt AL, Orrison WW, Yeo RA, et al. Clinical significance of MRI white matter lesions in the elderly. Neurology 1989;39:1470-4.

12 Tupler LA, Coffey CE, Logue PE, et al. Neuropsychological importance of subcortical white matter hyperintensity. Arch Neurol 1992;49:1248-52.

13 de Groot JC, de Leeuw F-E, Oudkerk M, et al. Cerebral white matter lesions and cognitive function: the Rotterdam scan study. Ann Neurol 2000;47:145-51.

14 Awad IA, Johnson PC, Spetzler RF, et al. Incidental subcortical lesions identified on magnetic resonance imaging in the elderly. II. Postmortem pathological correlations. Stroke 1986;17:1090-7.

15 Geschwind N. Disconnection syndromes in animals and man. Brain 1965;88:237-94.

16 Basser PJ, Pierpaoli C. Microstructural and physiological features of tissue elucidated by quantitative-diffusion-tensor MRI. J Magn Reson Ser B 1996;111:209-19.

17 Pierpaoli C, Basser PJ. Toward a quantitative assessment of diffusion anisotropy. Magn Reson Med 1996;36:893-906.

18 Makris N, Worth AJ, Sorensen AG, et al. Morphometry of in vivo human white matter association pathways with diffusion-weighted magnetic resonance imaging. Ann Neurol 1997;42:951-62.

19 Chabriat H, Pappata S, Poupon C, et al. Clinical severity in CADASIL related to ultrastructural damage in white matter: in vivo study with diffusion tensor MRI. Stroke 1999;30:2637-43.

20 O'Sullivan M, Summers PE, Jones DK, et al. Normal-appearing white matter in ischemic leukoaraiosis: a diffusion tensor MRI study. Neurology 2001;57:2307-10.

21 Bamford JM, Sandercock P, Dennis M, et al. Classification and natural history of clinically identifiable subtypes of cerebral infarction. Lancet 1991;337:1521-6.

22 Jones DK, Horsfield MA, Simmons A. Optimal strategies for measuring diffusion in anisotropic systems by magnetic resonance imaging. Magn Reson Med 1999;42:515-25.

23 Bland JM. An introduction to medical statistics, 3rd ed. Oxford: Oxford University Press, 2000.

24 Boone KB, Miller BL, Lesser IM, et al. Neuropsychological correlates of whitematter lesions in healthy elderly subjects: a threshold effect. Arch Neurol 1992;49:549-54.

25 Ylikoski R, Ylikoski A, Erkinjuntti T, et al. White matter changes in healthy elderly persons correlate with attention and speed of mental processing. Arch Neurol 1993;50:818-24.

26 Junque $C$, Pujol J, Vendrell $P$, et al. Leuko-araiosis on magnetic resonance imaging and speed of mental processing. Arch Neurol 1990;47:151-6.

27 Schmidt R, Fazekas F, Offenbacher H, et al. Neuropsychologic correlates of MRI white matter hyperintensities: a study of 150 normal volunteers. Neurology 1993;43:2490-4.

28 Breteler MMB, van Amerongen NM, VanSwieten JC. Cognitive correlates of ventricular enlargement and cerebral white matter lesions on magnetic resonance imaging: the Rotterdam Study. Stroke 1994;25:1109-15.

29 Schmidt R, Fazekas F, Kapeller P, et al. MRI white matter hyperintensities: three-year follow-up of the Austrian Stroke Prevention Study. Neurology 1999;53:132-9.

30 Yamauchi H, Fukuyama H, Ogawa M, et al. Callosal atrophy in patients with lacunar infarction and extensive leukoaraiosis. An indicator of cognitive impairment. Stroke 1994;25:1788-93.

31 Fox NC, Scahill RI, Crum WR, et al. Correlation between rates of brain atrophy and cognitive decline in AD. Neurology 1999;52:1687-9.

32 O'Brien JT, Paling S, Barber R, et al. Progressive brain atrophy on serial MRI in dementia with Lewy bodies, $A D$, and vascular dementia. Neurology 2001;56:1386-8.

33 Klingberg T, Hedehus $M$, Temple $E$, et al. Microstructure of tempero-parietal white matter as a basis for reading ability: evidence from diffusion tensor magnetic resonance imaging. Neuron 2000;25:493-500.

34 Basser PJ, Pajevic S, Pierpaoli C, et al. In vivo fiber tractography using DT-MRI data. Magn Reson Med 2000;44:625-32.

35 Lezak DM. The problem of assessing executive functions. Int J Psychol 1982;17:281-97. 